\title{
Violencia de género y autoestima de mujeres del centro poblado Huanja - Huaraz, 2017
}

Ericson Felix Castillo Saavedra 1,a, Janette Vanesa Bernardo Trujillo 2,b, Marleny Alida Medina Reyes 3,b

RESUMEN

Objetivo: Determinar la relación entre la violencia de género y el nivel de autoestima de las mujeres del centro poblado Huanja - Huaraz, 2017.

Materiales y métodos: Estudio cuantitativo, prospectivo, correlacional de corte transversal. La población muestral estuvo conformada por 55 mujeres registradas como habitantes del centro poblado Huanja, que cumplieron con los criterios de inclusión y exclusión de la investigación. Para el análisis y estudio de las variables se utilizaron instrumentos previamente validados con una confiabilidad de 0,81 para ambos cuestionarios.

Resultados: La violencia física representó el 38,2 \% de casos registrados, seguido de 29,1\%; 14,6\% y 18,1 \% para la violencia psicológica, sexual y económica respectivamente; y la autoestima presentó nivel bajo (52,8 \%), nivel medio $(43,6 \%)$ y nivel alto $(3,6 \%)$. El coeficiente de Correlación de Spearman ( $r s$ evidenció relación negativa y no significativa entre los tipos de violencia y la autoestima: violencia física ( $r s=-0,123 ; p=0,371)$, violencia psicológica ( $r s=-0,181 ; p=0,186)$, violencia sexual $(r s=-0,177 ; p=0,195)$ y violencia económica $(r s=-0,075 ; p=0,589)$.

Conclusiones: La violencia física que viven las mujeres del centro poblado de Huanja - Huaraz es la que se presenta con mayor frecuencia, por encima de la psicológica, sexual y económica. Las mujeres, víctimas de violencia, a través de su autoestima, se sienten frustradas, fracasadas y desvalorizadas ante la sociedad, por lo que, a mayor violencia de tipo física, psicológica, sexual y económica, menor autoestima.

Palabras clave: Violencia contra la mujer; violencia sexual; abuso físico; autoestima (Fuente: DeCS BIREME).

\section{Gender violence and self-esteem in women from the Huanja - Huaraz hamlet, 2017}

\section{ABSTRACT}

Objective: To determine the relationship between gender violence and level of self-esteem in women from the Huanja Huaraz hamlet, 2017.

Materials and methods: A quantitative, prospective, cross-sectional, correlation study. The sample population consisted of 55 women registered as inhabitants of the Huanja - Huaraz hamlet, who met the inclusion and exclusion criteria of the research. For the variables' analysis and study, previously validated instruments with a reliability of 0.81 were used for both questionnaires.

Results: Physical violence accounted for $38.2 \%$ of the recorded cases, followed by $29.1 \%, 14.6 \%$ and $18.1 \%$ for psychological, sexual and economic violence, respectively. Self-esteem was low in $52.8 \%$, moderate in $43.6 \%$, and high in $3.6 \%$. The Spearman's rank correlation coefficient ( $r s$ ) showed a negative and non-significant relationship between the types of violence and self-esteem: physical violence $(r s=-0.123, p=0.371)$, psychological violence $(r s=-0.181, p=0.186)$, sexual violence $(r s=-0.177, p=0.195)$ and economic violence $(r s=-0.075, p=0.589)$.

Conclusions: Physical violence experienced by women from the Huanja - Huaraz hamlet is the most frequent type of violence reported, followed by psychological violence, sexual violence and economic violence. Regarding their selfesteem, women victims of violence feel frustrated, unsuccessful and worthless before society. Therefore, the greater the physical, psychological, sexual and economic violence in women, the lower their self-esteem.

Keywords: Violence against women; sex offenses; physical abuse; self concept (Source: MeSH NLM).

1. Universidad Nacional de Trujillo. Trujillo, Perú.

2. Centro de Salud de Huanja. Huaraz, Perú.

3. Ministerio de Salud. Lima, Perú.

a. Químico Farmacéutico.

b. Obstetra. 


\section{INTRODUCCIÓN}

La violencia contra la mujer es uno de los problemas mundiales que afecta a todo tipo de sociedades, y va aumentando considerablemente en los últimos años, siendo un problema social preponderante a nivel mundial (1).

La Organización Mundial de la Salud (OMS) informó que, a nivel mundial, cerca de un tercio ( $30 \%$ ) de las mujeres que han tenido una relación de pareja habría sufrido violencia física y/o sexual. Según datos reportados en más de 80 países, se encontró que Japón tuvo el porcentaje más bajo sobre violencia de género (13\%), representando esta cifra un factor importante para frenar el desarrollo de un país $(2,3)$.

En el Perú se reportaron cifras elevadas sobre violencia física (61\%), mientras que, en el ámbito local en el año 2016, 4 de cada 10 mujeres ha sido víctima de violencia por parte de su pareja ${ }^{(4)}$. Asimismo, en un estudio realizado en Huancavelica por Ventura ${ }^{(5)}$, se encontraron niveles altos de violencia contra la mujer emprendedora (91\%), cifra muy elevada, que estaría representando la vulneración de los derechos humanos de la mujer.

La violencia de género se manifiesta de diversas formas como: violencia física, psicológica, sexual y económica, y aunque esta última no se da a conocer en gran medida, es una de las que ejerce mayor dominancia contra la mujer a lo largo de la historia, e incluso en la mujer moderna ${ }^{(5,6,7)}$.

La violencia de género se origina del comportamiento social aprendido desde los antepasados o por múltiples factores que permiten que se siga perpetrando, repercutiendo así en la salud y el bienestar físico y psicológico de la mujer. Analizado desde la perspectiva de la salud pública, genera daño no solo en la víctima en el momento de la agresión, sino en repercusiones traumáticas en ella y en los hijos, que no solamente lo perciben, sino también la presencian (3).

Por otro lado, todo tipo de violencia de género repercute directa o indirectamente en la autoestima, que disminuye a medida que pasa el tiempo y continúa con la convivencia; ya que la autoestima surge del entorno social que tiene la mujer a lo largo de su vida, tanto en el ámbito familiar y de pareja ${ }^{(8)}$.

La autoestima es la manera de valorarnos a nosotros mismos, ya sea en la forma como se ve o como se siente la mujer respecto a su apariencia, sus valores, sus defectos, sus habilidades, sus virtudes, la forma como actúa y como se siente valorada y estimada por las personas que la rodean. Proviene de las experiencias ocurridas y de los entornos en los que vivió, y se van desarrollando desde el nacimiento en función al amor, seguridad, sentirse valorado y ser capaz de realizar y cumplir sus metas $(8,9,10)$. Según algunos autores ${ }^{(10,11)}$, la autoestima se desarrolla en 5 componentes: autorrespeto, autoaceptación, autoevaluación, autoconcepto y autoconocimiento.

Es por ello que una mujer violentada posee una autoestima disminuida; se considera una mujer inútil, fracasada, que no puede dirigir a sus hijos y sin autocontrol en ella misma; incrementando el riesgo de problemas mentales, sin llegar necesariamente a impartir maltrato a sus hijos, pero si a tener múltiples riesgos sociales en su vida presente y futura ${ }^{(2,12,13)}$.

El estudio busca desarrollar evidencias de la realidad en nuestro país, debido a que en muchas ocasiones aquellas mujeres que viven en un entorno de violencia nunca lo mencionan o, peor aún, no lo consideran un problema; al igual que muchas autoridades de las zonas autóctonas de nuestra región, lo cual podría generar también el maltrato infantil y múltiples problemas sociales ${ }^{(12,14,15)}$.

El objetivo de este estudio fue determinar la relación entre la violencia de género y el nivel de autoestima de las mujeres del centro poblado de Huanja; proporcionando instrumentos de medición validados y confiables para ambas variables, lo cual generaría valiosos aportes en la comunidad científica.

\section{MATERIALES Y MÉTODOS}

El estudio fue cuantitativo, prospectivo, correlacional de corte transversal ${ }^{(16)}$. La población muestral estuvo constituida por 55 mujeres entre 18 y 64 años registrados en el Sistema de Vigilancia Comunitaria del Establecimiento de Salud de Huanja, que cumplieron con los criterios de inclusión y exclusión de la investigación.

Para la variable violencia de género se utilizó la técnica de la encuesta, y como instrumento un cuestionario tomado de Guerra y Morales ${ }^{(17)}$ y García ${ }^{(18)}$, modificado por la autora. Se utilizó la Escala Rensis Likert y se validó mediante juicio de experto a través de la metodología focus group. La confiabilidad correspondió a 0,81 según Alfa de Cronbach y consta de 24 ítems que abarcan 4 dimensiones: violencia física, con 6 ítems (nivel bajo con 6 puntos, medio entre 12 y 18 puntos y alto con 24 puntos); violencia psicológica, con 7 ítems (nivel bajo con 7 puntos, medio entre 14 y 21 puntos y alto con 28 puntos); violencia sexual, con 6 ítems (nivel bajo con 6 puntos, medio entre 12 y 18 puntos y alto con 24 puntos); y, violencia económica, con 4 ítems (nivel bajo con 4 puntos, medio entre 5 y 12 puntos y alto con 16 puntos). El puntaje general de la violencia de género fue: nivel bajo entre 23 a 38 puntos; medio, entre 39 a 56, alto entre 57 a 74 puntos y grave, entre 75 a 92 puntos. 
Para la variable autoestima se utilizó la técnica de la encuesta, y como instrumento un cuestionario tomado de Valenzuela ${ }^{(19)}$ y modificado por la autora. Se utilizó la Escala Rensis Likert y se validó mediante juicio de experto a través de la metodología focus group. La confiabilidad correspondió a 0,84 según Alfa de Cronbach y consta de 40 ítems que abarcan 5 dimensiones: autorrespeto, con 8 ítems (nivel bajo entre 8 a 16 puntos, medio entre 17 y 24 puntos y alto entre 25 a 32 puntos); autoaceptación, con 8 ítems (nivel bajo entre 8 a 16 puntos, medio entre 17 y 24 puntos y alto entre 25 a 32 puntos); autoevaluación, con 8 ítems (nivel bajo entre 8 a 16 puntos, medio entre $17 \mathrm{y}$ 24 puntos y alto entre 25 a 32 puntos); autoconcepto, con 8 ítems (nivel bajo entre 8 a 16 puntos, medio entre $17 \mathrm{y}$ 24 puntos y alto entre 25 a 32 puntos); autoconocimiento, con 8 ítems (nivel bajo entre 8 a 16 puntos, medio entre 17 y 24 puntos y alto entre 25 a 32 puntos). El puntaje general de la autoestima fue: nivel bajo entre 40 a 80 puntos; medio, entre 81 a 120; y alto, entre 121 a 160 puntos.

Los datos fueron analizados mediante estadísticas descriptivas. La relación entre violencia de género y autoestima fue evaluada mediante la Correlación de Spearman. Los datos fueron analizados mediante el programa SPSS, versión 24.0.

\section{RESULTADOS}

En el presente estudio se entrevistaron a 55 mujeres registradas en el centro poblado Huanja - Huaraz que cumplieron con los criterios de inclusión y exclusión, y que presentaron episodios de violencia en cualquiera de sus modalidades.

De acuerdo a los resultados obtenidos, en la tabla 1 se observó que el tipo más frecuente de violencia fue la física con $38,2 \%$; mientras que el $29,1 \% ; 14,6 \%$ y $18,1 \%$ correspondieron a la psicológica, sexual y económica respectivamente. En este sentido, los resultados obtenidos revelarían que la violencia de género surge del comportamiento social enseñado o por múltiples factores sociales que permiten la continuidad de este tipo de agresiones, repercutiendo de esta manera, en la salud y en el bienestar físico y psicológico de la mujer ${ }^{(20,21)}$ (Tabla 1).

Tabla 1. Tipo de violencia de las mujeres del centro poblado Huanja - Huaraz

\begin{tabular}{|lcc|}
\multicolumn{1}{c}{ Violencia de género } & F & $\%$ \\
\hline Física & 21 & 38,2 \\
Psicológica & 16 & 29,1 \\
Sexual & 8 & 14,6 \\
Económica & 10 & 18,1
\end{tabular}

Se evidenció que el $52,8 \%$ de las mujeres presentaron autoestima baja; mientras que para la autoestima media y baja correspondieron $43,6 \%$ y 3,6 \% respectivamente. De estos resultados se puede afirmar que una mujer violentada que posee autoestima disminuida, se considera una persona inútil, sin capacidades de surgir, sin poder dirigir a sus hijos ni a ellas mismas; además, incrementarían el riesgo de sufrir problemas psicológicos, que podrían conllevar a estados depresivos y a episodios de suicidio en los casos más graves (Tabla 2).

Por otro lado, una autoestima alta reflejaría en la vida de las personas, en especial en las mujeres, el propósito de consolidar su desenvolvimiento en la sociedad, respecto al aprendizaje, solución de problemas, decisiones asertivas en al ámbito familiar, social o laboral, en sentirse capacitado; así como tener la autonomía personal y la capacidad de tomar decisiones correctas; todo ello para el logro de un adecuado desarrollo humano ${ }^{(10,11)}$.

Tabla 2. Nivel de autoestima de mujeres del centro poblado Huanja - Huaraz

\begin{tabular}{|c|c|c|c|}
\hline Autoestima & $\mathbf{F}$ & $\%$ & * \\
\hline Baja & 29 & 52,8 & 9,3 \\
\hline Media & 24 & 43,6 & \\
\hline Alta & 2 & 3,6 & \\
\hline
\end{tabular}


Respecto al análisis de Spearman según las variables de estudio, se observó que existe relación negativa, pero no estadísticamente significativa entre la autoestima y los tipos de violencia, lo que indicaría que a medida que se incrementa la violencia física, psicológica, sexual y económica, la autoestima disminuye (Tabla 3).

Las mujeres con baja autoestima suelen experimentar ansiedad ante situaciones afectivas y de intimidad, y se debería a la dificultad de sentirse seguras y espontáneas en sus relaciones interpersonales. Asimismo, crean mecanismos de defensa como negación, intelectualización y fantasía, para poder protegerse de la amenaza frente a posibles rechazos sociales, incluso a críticas personales u opiniones que cuestionen sus creencias ${ }^{(14,15,20)}$.

Tabla 3. Coeficiente de correlación bivariada de Spearman para las variables de estudio

\begin{tabular}{lccccc}
\multicolumn{1}{c}{ Variable } & $\mathbf{1}$ & $\mathbf{2}$ & $\mathbf{3}$ & $\mathbf{4}$ & $\mathbf{5}$ \\
\hline $\begin{array}{l}\text { 1. Violencia física } \\
\text { P }\end{array}$ & 1 & & & & \\
2. Violencia psicológica & $-0,181$ & 1 & & & \\
P & 0,186 & & & & \\
3. Violencia sexual & $-0,177$ & 0,591 & 1 & & \\
P & 0,195 & 0,000 & & & \\
4. Violencia económica & 0,041 & 0,536 & 0,500 & 1 & \\
P & 0,001 & 0,000 & 0,000 & & \\
5. Autoestima & $-0,123$ & $-0,181$ & $-0,177$ & $-0,075$ & 1 \\
P & 0,371 & 0,186 & 0,195 & 0,589 & \\
& & & & & $\mathrm{n}=55$
\end{tabular}

\section{DISCUSIÓN}

En la relación de la autoestima y la violencia contra la mujer se encontró que a medida que se incrementa la violencia física, psicológica, sexual y económica, la autoestima es menor. Ruíz et al ${ }^{(22)}$ encontraron que el $24,8 \%$ de casos de violencia se debían a un nivel cultural y socioeconómico bajo. De la misma forma, Barja ${ }^{(23)}$ reportó en un nosocomio de Lima (Perú) porcentajes altos respecto a violencia física $(66,95 \%)$, violencia sexual $(65,53 \%)$, violencia psicológica $(64,77 \%)$ y violencia económica $(63,64 \%)$.

La violencia es un fenómeno multifactorial y multidimensional que provoca muchas consecuencias en diferentes aspectos de la vida, y principalmente si ocurre en la niñez y en la etapa escolar ${ }^{(18,19)}$.

En este sentido, Huanja (Huaraz) es una comunidad rural, en el cual no se tiene mayor acceso a la educación y economía, por lo que, las características del contexto estarían influyendo significativamente en el perfil del agresor. Al respecto, Ponce (4) menciona que 2 de cada 10 mujeres fueron víctimas de violencia por parte de sus parejas, manifestando que es más intenso en la zona rural, y reflejaría que en estas zonas existe una cultura de machismo, a diferencia de las zonas urbanas, en las que las mujeres tienen apoyo de instituciones para evitar y castigar los abusos de parte de sus parejas. También influye el factor socioeconómico y educativo que tiene cada una de ellas que las hace soportar todo tipo de violencia.

En lo que respecta al nivel de autoestima de las mujeres encuestadas se encontró que el $52,7 \%$ presenta autoestima baja, lo que demuestra que ellas no desarrollaron adecuadamente los componentes de la autoestima que se van formando desde la niñez, de acuerdo a lo que vive a diario y de su entorno. Morales et al. (24) refieren que las mujeres que han presentado episodios de violencia se sienten inútiles, creen que no sirven para nada, que son un fracaso, que no tienen motivos para sentirse orgullosas, y desean tener más respeto por ellas mismas. Por su parte, López et al. (7) expresan que los constantes maltratos recibidos por su cónyuge, hacen que las mujeres cambien de actitud, de estados de ánimo, forma de ser, sobretodo en el hogar ya sea con sus hijos o su cónyuge.

Algunos autores $(14,15,25)$ manifiestan que la formación de la autoestima se inicia desde el momento en que una persona es concebida, cuando el vínculo entre padres se consuma, comienza la carga de mensajes que recibe de manera energética y luego emocional. El organismo recibe en el vientre todas las manifestaciones y emociones a manera de energía; el cerebro produce una serie de compuestos químicos que se esparcen por todo el cuerpo y graba en 
su sistema nervioso en desarrollo, sin saber si es bueno o malo, y repercutirá en sus comportamientos futuros; por tanto, las piezas claves para el desarrollo de su autoestima son los padres y las figuras de autoridades que tendrá en casa.

La relación negativa y no estadísticamente significativa entre los tipos de violencia de género y la autoestima presentada por las mujeres del centro poblado Huanja (Huaraz), evidencia que uno de los factores que puede estar relacionado a este resultado encontrado, es el maltrato conyugal. Navarro (1) afirma que las mujeres casadas 0 convivientes que han presentado algún tipo de violencia, carecen principalmente de autoestima, por su alto grado de desvalorización, afecto personal y el hecho de asumir un alto grado de pasividad y sumisión, y expresaría una reciprocidad entre la violencia que existe contra la mujer y su autoestima dañada, a más violencia vivida mayor será la pérdida de la autoestima, sobre todo si se convive con el agresor.

La violencia contra la mujer por su pareja es la forma más frecuente y dañina, debido a que hay una convivencia diaria con su agresor, existiendo un lazo emocional que une a la mujer, el deber de soportar por sus hijos. Desde el punto de vista cultural, la violencia está enmarcada por una estructura patriarcal, como es que los varones son superiores, por tanto, tienen el derecho y la responsabilidad de imponer medidas correccionales como una forma de control de la mujer en cuanto a su conducta (24,26).

Las víctimas de violencia de género, además de tener un sufrimiento físico y mental, pueden sentirse incapaces de hablar de lo que les pasa con otros familiares o amigos por miedo o por sentirse culpables de que esto les suceda, imposibilitándoles cambiar la situación, ya sea por causa de dependencia económica o por sentirse incapaces de escapar de la relación en el que se encuentran ${ }^{(2,5,7,8)}$.

En conclusión, la relación entre los tipos de violencia y el nivel de autoestima de las mujeres del centro poblado de Huanja (Huaraz) fue negativa, por lo que, a mayor violencia de tipo física, psicológica, sexual y económica, menor autoestima.

Contribuciones de autoría: EFCS, JVBT y MAMR participaron en la concepción y diseño del estudio, recolección de datos, análisis e interpretación de datos, redacción del artículo, revisión crítica del artículo y aprobación de la versión final.

\section{REFERENCIAS BIBLIOGRÁFICAS}

1. Navarro Oliva E. Violencia de género, autoestima y consumo de alcohol en mujeres [tesis de postgrado]. Nuevo León:
Universidad Autónoma de Nuevo León; 2011.

2. Morales-Reyes A, Alonso-Castillo M, López-García K. Violencia de género y autoestima de las mujeres de la Ciudad de Puebla. Rev Sanid Milit Mex. 2011; 65(2): 48-52. Disponible en: http://www.medigraphic.com/cgi-bin/new/resumen. cgi?IDARTICULO=40287

3. Organización Mundial de la Salud. Estudio multipaís de la OMS sobre salud de la mujer y violencia doméstica [Internet]. Ginebra: OMS; 2005 [acceso 15 diciembre 2017]. Disponible en: http://apps.who.int/iris/ bitstream/10665/43390/1/924359351X_spa.pdf

4. Ponce Gómez J. Niveles de violencia contra la mujer emprendedora en el Perú: Un análisis basado en ENDES 2011. Revista de Ciencias Empresariales de la Universidad de San Martín de Porres. 2012; 3(2): 7-56. Disponible en: http:// www.administracion.usmp.edu.pe/wp-content/uploads/ sites/9/2014/02/sme_v3n21.pdf

5. Ventura Huaman R. Niveles de violencia contra las mujeres en las asociaciones de artesanía textil en Huancavelica, en el año 2014. Revista de Ciencias Empresariales de la Universidad de San Martín de Porres 2015; 6(2): 96-106. Disponible en: http://www.repositorioacademico.usmp.edu.pe/bitstream/ usmp/1749/1/sme_v6n2_art5.pdf

6. Carneiro L, Teodoro G, Chicone G. Autoestima de las mujeres que sufrieron violencias. Rev Latino-am Enfermagem. 2006; 14(5): 1-7. Disponible en: http://www.scielo.br/pdf/rlae/ v14n5/es_v14n5a09.pdf

7. López Angulo L, Apolinaire Pennini JJ, Array M, Moya Avila A. Autovaloración en mujeres víctimas de violencia de pareja. Medisur. 2006; 4(1): 9-12. Disponible en: http://www. medisur.sld.cu/index.php/medisur/article/view/171

8. Mendoza Flores ME, Martínez Hernandez G, Pizano Zarate $M L$, Lartigue Becerra T. Violencia de género, embarazo y autoestima en un área urbana de la ciudad de México. Rev Enferm Herediana. 2008; 1(1): 40-47. Disponible en: http:// faenf.cayetano.edu.pe/images/pdf/Revistas/2008/enero/ v1n1ao6.pdf

9. Espinoza Campoverde M. Maltrato conyugal y autoestima en mujeres que trabajan en los mercados de la Ciudad de Loja, Ecuador-2014 [tesis de postgrado]. Loja: Universidad Peruana Unión; 2014.

10. Sánchez A, Marcelino C. Fortalecimiento de la autoestima mediante la psicoterapia Gestalt en estudiantes del primer ciclo de una universidad privada de Lima metropolitana [tesis de postgrado]. Lima: Universidad Nacional Mayor de San Marcos; 2016.

11. Rodas Giron A. Relación entre autoestima y compromiso organizacional en docentes de una institución educativa [tesis de pregrado]. Guatemala: Universidad Rafael Landívar; 2013.

12. Ochoa O, Restrepo D, Salas Zapata C, Sierra G, Torres Y. Relación entre antecedente de maltrato en la niñez y comportamiento maltratador hacia los hijos. Itaguií, Colombia, 2012-2013. Rev colomb psiquiat. 2017; 30(20): 3034. Disponible en: https://www.sciencedirect.com/science/ article/pii/S0034745017300707

13. Suelves J, Jané M, Plasencia A. Violencia del compañero íntimo contra la mujer: una mirada desde la Salud Pública. Rev Esp Med Legal. 2010; 36(3): 98-103. Disponible en: http://www.elsevier.es/es-revista-revista-espanolamedicina-legal-285-articulo-violencia-del-companero-intimocontra-S0377473210700377 
14. Santandreu $M$, Torrents $L$, Roquero R, Iborra A. Violencia de género y autoestima: efectividad de una intervención grupal. Apuntes de Psicología, 2014; 32(1): 57-63. Disponible en: http://www.apuntesdepsicologia.es/index.php/revista/ article/view/487

15. Schnaas M, Ruíz Gonzales A, Juárez Miranda B. Asociación entre violencia y autoestima con respecto a las distintas etapas de vida en la mujer. Rev Mex Invest Psic. 2009; 1(1): 111-22. Disponible en: http://www. revistamexicanadeinvestigacionenpsicologia.com/article/ view/96

16. Hernández R, Fernández C, Baptista P. Metodología de la investigación. 6ed. México: McGraw-Hill, 2014.

17. Guerra G, Morales E. Violencia en Mujeres de una zona suburbana [tesis de pregrado]. Coatzacoalcos: Universidad Veracruzana; 2009.

18. García G, Guilly N. Antecedente de violencia doméstica y actitud violenta en hombres residentes en Manchay, Lima [tesis de pregrado]. Lima: Universidad Ricardo Palma; 2013.

19. Valenzuela S. Violencia intrafamiliar como factor de riesgo en la mujer embarazada y su producto [tesis de postgrado]. Colima: Universidad de Colima; 2004.

20. Henao Escobar J. La prevención de la violencia: una revisión temprana de programas y modalidades de intervención. Univ Psychol. 2005; 4 (2): 161-77. Disponible en: http://www. redalyc.org/html/647/64740205/

21. Doménech $M$, Iñiguez $L$. La construcción social de la violencia. Athenea. 2002; 2(1): 1-10. Disponible en: http: / / atheneadigital.net/article/viewFile/n2-domenechiniguez/54-pdf-es

22. Ruiz-Perez I, Escriba-Aguir V, Montero-Piñar I, Vives-Cases C, Rodríguez-Barranco M. Prevalence of intimate partner violence in Spain: A national cross-sectional survey in primary care. Aten Primaria. 2016; 3(2): 1-9. Disponible en: https:// www.ncbi.nlm.nih.gov/pubmed/27394929

23. Barja Ore J. Factores que influyen en la violencia basada en género en mujeres de 20 a 40 años atendidas en el consultorio externo de ginecología del Hospital Sergio Bernales - Comas, noviembre - diciembre 2015 [tesis de preg rado]. Lima: Universidad Nacional Mayor de San Marcos; 2016.

24. Morales-Reyes A, Alonso-Castillo M, López-Garcia K. Violencia de Género y autoestima de las mujeres de la Ciudad de Puebla. Rev Sanid Milit Mex. 2011; 65(2): 48-52. Disponible en: http://www.medigraphic.com/cgi-bin/new/resumen. cgi?IDARTICULO $=40287$

25. Uribe Castro M. Relación que existe entre el nivel de autoestima y el tipo de dinámica familiar de adolescentes del Centro Educativo Leoncio Prado, San Juan de Miraflores, 2011 [tesis de pregrado]. Lima: Universidad Nacional Mayor de San Marcos; 2012.

26. Nájera Hernandez C. Violencia de género en el contexto de pareja y su relación con la autoestima en el centro de desarrollo Integral de la familia, Arandas-México, 2013 [tesis de postgrado]. Arandas: Universidad Peruana Unión; 2013.

Fuentes de financiamiento:

Este artículo ha sido financiado por los autores.

Conflictos de interés:

Los autores declaran no tener ningún conflicto de interés.

\section{Correspondencia:}

Ericson Felix Castillo Saavedra

Dirección: Av. Juan Pablo II S/N. Trujillo, Perú.

Teléfono: 979127933

Correo electrónico: ericson_fcs@hotmail.com

Recibido: 26 de diciembre de 2017 Evaluado: 28 de diciembre de 2017 Aprobado: 06 de febrero de 2018

(C) La revista. Publicado por Universidad de San Martín de Porres, Perú. (cc) ву Licencia de Creative Commons Artículo en acceso abierto bajo términos de Licencia Creative Commons Atribución 4.0 Internacional. (http: //creativecommons.org/licenses/by/4.0/)

ORCID iDs

Ericson Felix Castillo Saavedra: (Dhttps: //orcid.org/0000-0002-9279-7189 Janette Vanesa Bernardo Trujillo: Dhttps: / / orcid.org/0000-0002-6054-5219 Marleny Alida Medina Reyes: iD https: //orcid.org/0000-0001-7777-0538 